\title{
Dam Wall Detection and Tracking Using a Mechanically Scanned Imaging Sonar
}

\author{
Wajahat Kazmi, Pere Ridao, David Ribas and Emili Hernández
}

\begin{abstract}
In Dam inspection tasks, an underwater robot has to grab images while surveying the wall meanwhile maintaining a certain distance and relative orientation. This paper proposes the use of an MSIS (Mechanically Scanned Imaging Sonar) for relative positioning of a robot with respect to the wall. An imaging sonar gathers polar image scans from which depth images (Range \& Bearing) are generated. Depth scans are first processed to extract a line corresponding to the wall (with the Hough Transform), which is then tracked by means of an EKF (Extended Kalman Filter) using a static motion model and an implicit measurement equation associating the sensed points to the candidate line. The line estimate is referenced to the robot fixed frame and represented in polar coordinates $(\rho \& \theta)$ which directly corresponds to the actual distance and relative orientation of the robot with respect to the wall. The proposed system has been tested in simulation as well as in water tank conditions.
\end{abstract}

\section{INTRODUCTION}

This research was performed in the context of a project funded by the Spanish Ministry of Science and Technology which aims to explore the possible industrial applications of underwater robots such as dam, harbour and underwater cable/pipe inspection. After a meeting with the civil engineers of the ENDESA power generation company in Spain, one of the tasks identified for dam inspection was a visual survey of the wall in order to assess the state of the concrete and/or the fence which protects the water inlet of the penstock gallery(an intake structure that controls water flow). Until now, these inspections were commonly achieved through the visualization of a video recorded by a professional diver without using any geo-referencing information.

Although, over the last few years, several companies have claimed providing underwater robots for dam inspection, to the best of the author's knowledge, none of them provides an integral solution to the problem. Normally they propose the use of small class ROVs (Remotely Operated Vehicles), working as teleoperated cameras for video recording, to replace the professional diver who traditionally occupied this place. There exist few research precedents providing an added value solution. One of the most relevant works is the ROV3 system developed by the researchers of the Institut de recherche HydroQuébec (Canada) [1]. This is a small ROV, localized through an LBL (Long Base Line) system, which makes use of a multibeam sonar for collision avoidance. The

This research was sponsored by the European Commission through Erasmus Mundus Scholarship (VIBOT Masters, http://www.vibot.org) program.

The authors are with the Department of Computer Engineering, University of Girona, 17071 Girona, Spain.

wajahat.kazmidlive.com, peredeia.udg.edu, dribas@eia.udg.edu, emilihbeeia.udg.edu system is able to control the distance to the wall and includes several video cameras as well as a laser system for $2 \mathrm{D}$ and 3D measurements. The COMEX and the Electricité De France companies (France) developed a similar project [2]. In this case, an ROV manufactured by COMEX was localized using a 5-transponder LBL. Again, several video cameras together with 2D (double spot) laser system were used to take measurements. During the year 2002, in collaboration with the Research Development and Technological Transfer Centre (CIFATT) IPA-Cluj, our team used the URIS robot working as an ROV to build an image mosaic [3] of a small area of the wall of the Tartina Dam in the surroundings of Cluj (Romania). To the best of the author's knowledge, this was the first time that image mosaicking techniques were applied for dam inspection. An image mosaic gives an added advantage as it provides the civil engineers with a global view of the inspection area. Unfortunately, the ROV was not localized and hence the resulting image mosaic was not georeferenced.

Our current approach (Fig.1) for obtaining a georeferenced, high resolution visual map of the wall, consists of programming the ICTINEU ${ }^{A U V}$ [4] robot to follow a pre-defined survey path in front of a selected area of the wall while being localized using a dead reckoning system based on a DVL(Doppler Velocity Log), a heading and a depth sensor. To bound the drift, a buoy equipped with a DGPS (Differential Global Positioning System), an MRU (Motion Reference Unit) and a USBL(Ultra Short Base Line) is used which provides geo-referenced position fixes, feed to the robot through an umbilical cable when available or through an acoustic modem otherwise. While moving along the wall, the robot gathers images of the wall tagged with a time stamp and synchronized with all the sensor data including the navigation. As discussed in [5], in tasks such as dam survey, it is important to maintain a specified distance and specially a certain relative orientation with respect to the wall (usually orthogonal) in order to achieve a high resolution image mosaic. A similar problem was faced for ship hull inspection as reported in [6]. In that case, the DVL was looking towards the ship's hull and the range readings were used to compute the relative orientation of the robot with respect to the hull, while in our case, the robot is passively stable in roll and pitch and multiple range and bearing measurements of sonar are used to compute the robot's relative orientation and distance in front of the dam wall.

The aim of this paper is to propose, design and test, a suitable method to detect the wall of a dam, its distance and 


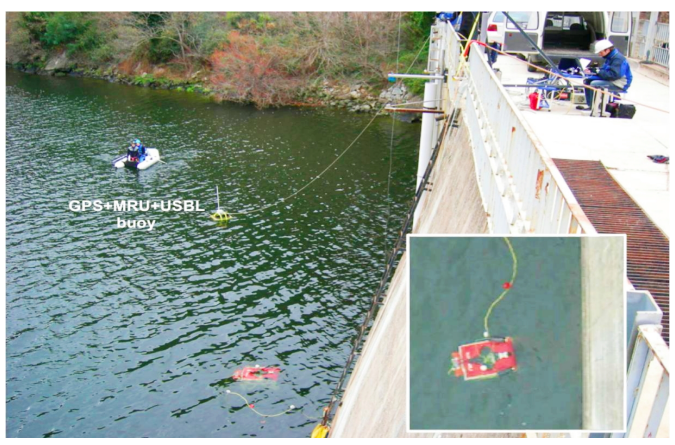

Fig. 1. AUV orientation problem in a dam's wall inspection task

orientation with respect to the robot. Although not discussed in this paper, these parameters will further be used for close loop control in order to maintain a relative position during the survey. The proposed system works by first acquiring an acoustic image of a specified sector of the wall in front of the robot with an MSIS. Then, that acoustic image is transformed into a range \& bearing scan which is a set of points compounded with the robot trajectory representing it in an initial frame of reference. After this pre-processing, a line feature is estimated by means of a standard Hough Transform but using an improved sonar model to decide which candidate line should be voted for by each sonar point. In order to further utilize sonar data for correcting this estimate of the wall, the estimated line is used to initialize an EKF based on a static motion model. Updates for every compatible sonar point of the scan are carried out using an implicit measurement equation stating that the point belongs to the line. The system has been tested in simulation as well as in water tank conditions. Finally, different alternatives are studied and compared in order to improve the tracking such as using heading updates from compass or complete navigation information from DVL to reference the sonar data to a selected frame of reference.

The paper is organized as follows. First, the sensor suite needed onboard is described in section II. Section III is devoted to the description of the feature extraction and tracking. Finally the simulation environment and the results are reported in sections IV and V respectively, before the conclusions in section VI.

\section{SENSOR Suite}

\section{A. MiniKing Imaging Sonar (MSIS)}

The MiniKing Imaging Sonar is a compact device designed for use in underwater applications such as obstacle avoidance and target recognition for both AUVs and ROVs. This sonar can perform scans in a $2 \mathrm{D}$ plane by rotating a fanshaped sonar beam through a series of small angle steps. It can be programmed to cover variable sector lengths starting from a few degrees to a full $360^{\circ}$ scan. In this project, it is programmed for a $90^{\circ}$ sector, repetitive scan. Its fan-shaped beam has a vertical aperture angle of $40^{\circ}$ and a narrow horizontal aperture of $3^{\circ}$.

\section{B. Sontek Argonaut DVL}

The SonTek Argonaut Doppler Velocity Log is a sensor specially designed for AUV applications which measures ocean currents, vehicle speed over ground and through water using a precise 3-axis measurement system based on the Doppler shift effect. It operates at a frequency of $1500 \mathrm{kHz}$ and has a range of about $15 \mathrm{~m}$. The velocity information by this DVL is used to localize the robot through dead reckoning.

\section{Feature Extraction AND Tracking}

For planar structures such as walls, the intersection of the fan beam of the imaging sonar produces a 2D slice with high intensity values near the center. When the sonar beam rotates, this slice extends over the wall and forms a thick line (Fig.2). Segmenting this thick line produces a relatively concentrated zone of points which can safely be estimated to a line [7]. Although dam walls are generally curved to improve the structural resistance to the water pressure, generally, they can be accurately represented by a line feature. This line can be initially estimated applying the Hough Transform to a depth scan obtained after some pre-processing [8]. An improved sonar model is used to decide a set of compatible lines that a particular sonar-point-measurement should vote for. Then, the initial line candidate computed can further be refined in a tracking process where the points extracted from the sonar data are associated to the line estimate if a compatibility test is satisfied.

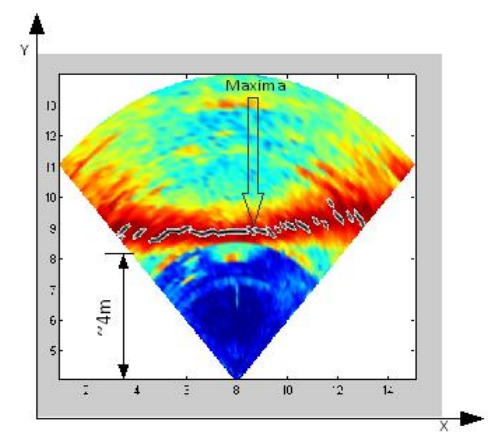

Fig. 2. Sonar Image (facing wall at $\approx 4 \mathrm{~m}, 90^{\circ}$ sector, single scan)

\section{A. Beam Segmentation and Range Detection}

The log file generated by the MSIS contains one row of data for every beam, with columns 4 to 503 containing echo intensities of a return. Keeping in mind the reflections from robot body itself along with reverberations from side and back walls, data from first and last few (around ten) columns are discarded. Maximum value of intensity for every row (or beam) is then taken as measurement corresponding to the features in the environment. In this work, the range of the sonar sensor is kept at $10 \mathrm{~m}$ with a resolution of $10 \mathrm{~cm}$. This means every column index from 4 to 503 of the log file represents intensity at a range $=($ index -3$) \times 10 \mathrm{~cm}$ 


\section{B. Sonar Model}

Due to the horizontal aperture (beamwidth), a bearing cannot be related to a single point in space. Leonard-Whyte [9] and Neira et al. [10] proposed a sonar model where a sonar measurement $\left(\rho^{S}, \theta^{S}\right)$ is not associated with a unique point but with an arc of points with radius $\rho^{S}$ and an aperture $\alpha$ being centered at the actual sonar measurement. Hence the points $\left(\rho_{j}^{S}, \theta_{j}^{S}\right)$ associated with the measurement are those satisfying:

$$
\theta^{S}-\alpha / 2 \leq \theta^{S_{j}} \leq \theta^{S}+\alpha / 2 \quad ; \quad \rho_{j}^{S}=\rho^{S}
$$

This extends the sonar measurement with a range of possible bearings within the horizontal aperture $\alpha$ of the beam (where $\alpha$ in our case is $3^{\circ}$ ). Hence, the set of candidate lines which explain the sonar measurement are those tangent to the corresponding arc. Although this model is suitable for mobile robots using wide angle low resolution sonar beams, it still needs to be improved to explain the behavior experimentally observed with the MSIS. For this reason, Ribas et al [8] further extended this model for beams with a narrow horizontal aperture and working underwater. In this case, the measurements are not only associated to the arc tangent surfaces within the horizontal aperture, but also to the surfaces incident at certain angles $\beta$ (for the Miniking sonar, $\left.\beta=60^{\circ}[8]\right)$. Now a candidate line $\left(\theta_{k}^{B}, \rho_{k}^{B}\right)$ is described by the following equations (see Fig.3)

$$
\begin{gathered}
\theta^{s j}-\beta / 2 \leq \theta_{k}^{B} \leq \theta^{s j}+\beta / 2 \\
\rho_{k}^{B}=x_{s j} \cos \theta_{k}^{B}+y_{s j} \sin \theta_{k}^{B}+\rho_{s j} \cos \theta_{k}^{S j}
\end{gathered}
$$

The base frame $\{B\}$ shown in Fig.3 is usually the vehicle

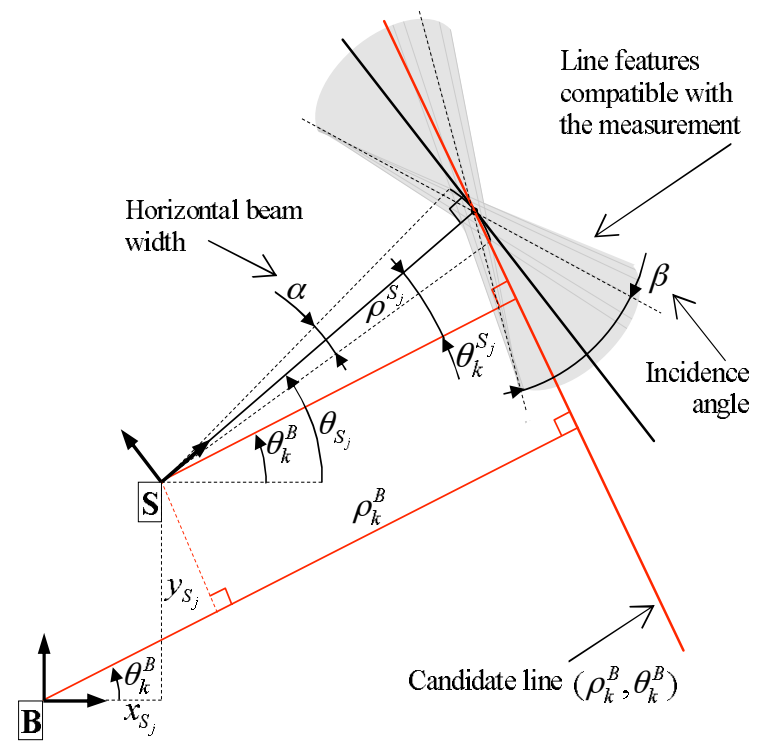

Fig. 3. Modified Sonar Model

frame and $\{S\}$ is the beam frame located and rotating with the transducer head.

\section{Tracking}

As explained above, the first scan is processed by means of the Hough Transform to produce an initial estimate of the line $\left(\rho_{L}, \theta_{L}\right) \equiv\left(\theta_{k}^{B}, \rho_{k}^{B}\right)$ which is then used to initialize the state vector of the EKF for tracking the line:

$$
x_{0}=\left[\begin{array}{c}
\rho_{L} \\
\theta_{L}
\end{array}\right] \quad ; \quad P_{0}=\left[\begin{array}{cc}
\sigma_{\rho}^{2} & 0 \\
0 & \sigma_{\theta}^{2}
\end{array}\right]
$$

Although, as stated in [11], it is possible to obtain an estimate of the covariance of the line feature through a careful processing of the acoustic imprint left by the line in the segmented image, in this work, for the sake of simplicity, the covariance has been initialized according to the resolution of the Hough Table. Hence the robot will move exploring the dam while the line representing the wall will remain almost static in the robot frame except for minor oscillations due to an imprecise control as well as some possible perturbations. Therefore, a static motion model including Gaussian noise to deal with these perturbations is used in the motion prediction step of the EKF:

$$
x_{k}=x_{k-1}+w_{k} \quad ; \quad E\left[w_{k}\right]=0 \quad ; \quad E\left[w_{k} w_{j}^{T}\right]=\delta_{k j} Q_{k}
$$

The updates of the line estimate come from the sonar-pointmeasurements:

$$
\begin{gathered}
\hat{s}_{k}=\left[\begin{array}{c}
\rho_{p} \\
\theta_{p}
\end{array}\right] \quad ; \quad s_{k}=\hat{s}_{k}+v_{k} \\
E\left[v_{k}\right]=0 ; \quad E\left[v_{k} v_{j}^{T}\right]=\delta_{k j} R_{k}
\end{gathered}
$$

where $R_{k}$ corresponds to the uncertainty of the sonar point measurement. In order to relate the points to the line, an implicit measurement equation is used [12] which states that the point belongs to the line, or equivalently, the point to line distance is zero:

$$
\begin{gathered}
h_{k}=h\left(q_{k}, x_{k}\right)=\rho_{L}-x_{p} \cos \theta_{L}-y_{p} \sin \theta_{L}=0 \\
q_{k}=q\left(z_{k}\right)=\left[\begin{array}{l}
x_{p} \\
y_{p}
\end{array}\right]=\left[\begin{array}{l}
\rho_{p} \cos \theta_{p} \\
\rho_{p} \sin \theta_{p}
\end{array}\right]
\end{gathered}
$$

where, for the sake of simplicity $\{B\}$ and $\{S\}$ origins have been considered coincident. Only the points which are statistically compatible with the line are used for the update. Individual compatibility for every point is checked using the compatibility test based on the Mahalanobis Distance:

$$
\begin{gathered}
D^{2}=h_{k}\left(H_{k} P_{k}^{-} H_{k}^{T}+V_{k} S_{k} V_{k}^{T}\right)^{-1} h_{k}^{T}<\chi_{d, \alpha^{\prime}}^{2} \\
S_{k}=J_{q_{k}} R_{k} J_{q_{k}}^{T} \\
J_{q_{k}}=\frac{\partial q_{k}}{\partial z_{k}} ; H_{k}=\frac{\partial h_{k}\left(q_{k}, x_{k}\right)}{\partial x_{k}} ; V_{k}=\frac{\partial h_{k}\left(q_{k}, x_{k}\right)}{\partial q_{k}}
\end{gathered}
$$

where $S_{k}$ is the measurement uncertainty in Cartesian coordinates, $D^{2}$ is the Mahalanobis Distance and $\chi_{d, \alpha^{\prime}}^{2}$ is the Chi Square value of confidence; $\alpha^{\prime}$ being the confidence level and $d$ the dimension of $h$ ( 1 in this case). If the point is compatible, the update is carried out using the EKF equations:

$$
K_{k}=P_{k}^{-} H^{T}\left(H_{k} P_{k}^{-} H_{k}^{T}+V_{k} S_{k} V_{k}^{T}\right)^{-1}
$$




$$
\begin{gathered}
\hat{x}_{k}=x_{k}^{-}-K_{k} h_{k} \\
\hat{P}_{k}=\left(I-K_{k} H_{k}\right) P_{k}^{-}
\end{gathered}
$$

\section{Integrating Heading Measurements}

Heading sensors, compass and/or gyros, are commonly available in ROVs and AUVs and can be used to further improve the line tracking. If the absolute orientation of the robot $\psi_{R}^{W}$, as well as the relative orientation of the wall with respect to the robot $\theta_{L}^{R}$ are known, it is possible to compute the absolute orientation of the dam $\beta_{L}^{W}$ :

$$
\beta_{L}^{W}=\psi_{R}^{W}+\theta_{L}^{R}
$$

Hence, after the first scan, once the line has been estimated,

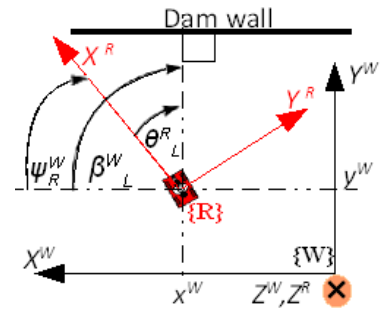

Fig. 4. Integrating Compass updates into EKF state vector

the EKF can be initialized including $\beta_{L}^{W}$ within the state vector:

$$
x_{0}=\left[\begin{array}{c}
\rho_{L}^{W} \\
\theta_{L}^{W} \\
\beta_{L}^{W}
\end{array}\right] ; P_{0}=\left[\begin{array}{lrr}
\sigma_{\rho}^{2} & 0 & 0 \\
0 & \sigma_{\theta}^{2} & \sigma_{\theta}^{2} \\
0 & \sigma_{\theta}^{2} & \left(\sigma_{\theta}^{2}+\sigma_{\psi}^{2}\right)
\end{array}\right]
$$

where it has been assumed that the robot was static enough during the first scan to consider $\theta_{L}^{R}$ independent from $\psi_{R}^{W}$ so:

$$
\sigma_{\beta}^{2}=\sigma_{\theta}^{2}+\sigma_{\psi}^{2} ; \sigma_{\beta \psi}=\sigma_{\theta}^{2}
$$

Since a linear observation equation relates the heading measurement with the state vector:

$$
\begin{gathered}
z_{k}=\psi_{R}^{W} ; \quad z_{k}=H x_{k}+v_{k} \\
E\left[v_{k}\right]=0 ; \quad E\left[v_{k} v_{j}^{T}\right]=\delta_{k j} R_{k}
\end{gathered}
$$

whenever a heading measurement is available, the following $H$ matrix:

$$
H=\left[\begin{array}{lll}
0 & -1 & 1
\end{array}\right]^{T}
$$

can be used together with the standard KF linear update equations to correct the current estimate. In case both measurements (the sonar-point and the heading) are available at same time step, the measurement vector becomes:

$$
q_{k}=\left[\begin{array}{c}
\rho_{p} \\
\theta_{p} \\
\psi_{R}^{W}
\end{array}\right]=\left[\begin{array}{c}
q\left(s_{k}\right) \\
\psi_{R}^{W}
\end{array}\right]
$$

and the observation equation becomes the following nonlinear vector function:

$$
h\left(q_{k}, x_{k}\right)=\left[\begin{array}{c}
\rho_{L}-x_{p} \cos \theta_{L}-y_{p} \sin \theta_{L} \\
\psi_{R}^{W}
\end{array}\right]
$$

In this case, the update is carried out as follows:

$$
\begin{gathered}
K_{k}=P_{k}^{-} H_{k}^{T}\left[H_{k} P_{k}^{-} H^{T}+V_{1_{k}} S_{k} V_{1_{k}}^{T}+V_{2_{k}} R_{k} V_{2_{k}}^{T}\right] \\
\hat{x}_{k}=x_{k}^{-}+K_{k}\left[z_{k}-h\left(q_{k}, x_{k}^{-}\right)\right] ; z_{k}=\left[\begin{array}{c}
0 \\
\psi_{R}^{W}
\end{array}\right]
\end{gathered}
$$

where,

$$
\begin{gathered}
H_{k}=\frac{\partial h_{k}\left(q_{k}, x_{k}\right)}{\partial x_{k}} \\
V_{1_{k}}=\frac{\partial h_{k}\left(q_{k}, x_{k}\right)}{\partial\left(\rho_{p}, \theta_{p}\right)} ; V_{2_{k}}=\frac{\partial h_{k}\left(q_{k}, x_{k}\right)}{\partial \psi_{R}^{W}}
\end{gathered}
$$

\section{E. Navigation Aid}

When available, the robot position can be used to improve the results. First, during the line detection phase, the sonar points must be compounded with the robot position otherwise, the acoustic image gets distorted due to the motion of the robot during the scanning process. For this purpose, all the sonar data is first referenced to a specific reference frame before the Hough Transform is used (in conjunction with the sonar model) to estimate the line. Second, during the tracking, the sonar points are also compounded with the the robot pose prior to the update of the line estimated with the EKF. It is worth noting that both, the points and the line, must be referenced to the same frame. Moreover, since navigation data often drifts due to the dead reckoning process, the reference frame must be changed periodically. In case no navigation data is available which is normally the case when an ROV is used, the robot must be static during the line detection and moving smoothly when the tracking. Navigation data plays a particularly important role in the presence of strong perturbations since, in this case, the static motion model for the line, expressed in the robot local frame, does not hold anymore while still applicable in an external global frame. Moreover, in this case, still it is possible to run periodically the Hough based line detector to reset the EKF, to avoid the drift of the tracking process.

A simple simulation is set up in MATLAB to exhaustively test the proposed algorithms prior to real testing with the AUV. For this purpose, synthetic data is generated for a virtual pool corresponding to the water tank dimensions used for field experiments

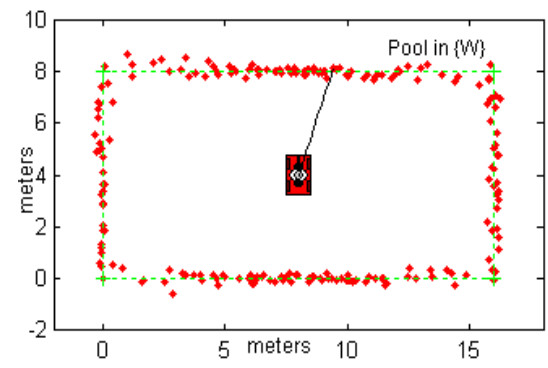

Fig. 5. Synthetic data displayed in $\{\mathrm{W}\}$ 


\section{F. Robot Motion Model}

Robot motion is simulated using a 3DOF (surge, sway and yaw) constant velocity kinematic model with acceleration noise:

$$
\left[\begin{array}{l}
x \\
y \\
\psi
\end{array}\right]_{k}^{W}=\left[\begin{array}{c}
x \\
y \\
\psi
\end{array}\right]_{k-1}^{W}+R_{R}^{W}\left[\left[\begin{array}{c}
u \\
v \\
r
\end{array}\right]_{k-1}^{R} \Delta t+\left[\begin{array}{c}
w_{\dot{u}} \\
w_{\dot{v}} \\
w_{\dot{r}}
\end{array}\right]_{k-1}^{R} \frac{\Delta t^{2}}{2}\right]_{(28)}
$$

where $\{W\}$ is world fixed frame located at the corner of the water tank, $\{R\}$ is the robot fixed frame and $R_{R}^{W}$ is the rotation matrix from $\{\mathbf{R}\}$ to $\{\mathrm{W}\}$. The vector $(x, y, \psi)^{W}$ corresponds to the robot pos in the world fixed frame while $(u, v, r)^{R}$ and $\left(w_{\dot{u}}, w_{\dot{v}}, w_{\dot{r}}\right)^{R}$ are the robot velocities and the acceleration noise respectively, both referenced to the robot frame. The robot is initialized to be at a desired position with some velocity. By changing the acceleration noise it is possible to carry out experiments where the robot remains static or it hovers around a specified spot at varying velocities.

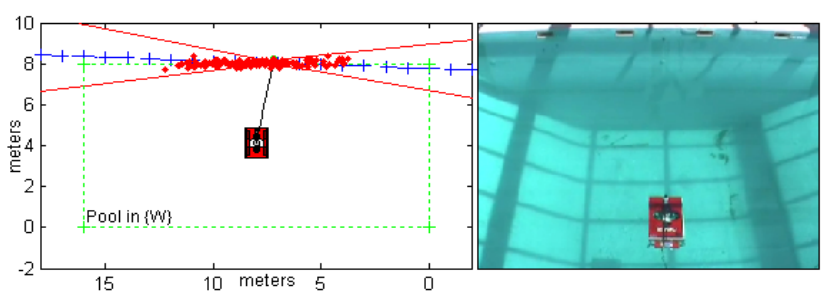

Fig. 6. Simulated Vs. Real environments

\section{Simulation EnVironment}

\section{A. Sonar Simulation}

The sonar beam is simulated as a line, with its origin in the transducer head, rotating at a certain speed to sweep a sector scan, repetitively. The sonar points are computed by the intersection between the sonar line and the walls of the rectangular water tank. The points are then corrupted in $\rho_{p}, \theta_{p}$ with Gaussian noise (Fig. 5).

\section{RESUlts}

In this section simulations as well as experiments using $I C T I N E U^{A U V}$ robot in the water tank of the Underwater Robotics Research Center (CIRS) of the universitat de Girona are described. In both cases, results with and without navigation aid are reported and discussed.

\section{A. Simulations}

Several simulations were carried out with synthetic data. A representative one is reported here. During the experiment, the robot was moving with an initial velocity $(u, v, r)^{R}=$ $(0.3 \mathrm{~cm} / \mathrm{s},-0.4 \mathrm{~cm} / \mathrm{s},-0.02 \mathrm{rad} / \mathrm{s})_{k}^{R}$ and acceleration noise $\left(\sigma_{\dot{u}}, \sigma_{\dot{v}}, \sigma_{\dot{r}}\right)_{k}^{R}=(0.0008,0.0008,0.008)_{k}^{R} \mathrm{~m} / \mathrm{s}^{2}$. Zero mean Gaussian noise with variances $\sigma_{\rho}^{2}=(30 \mathrm{~cm})^{2}$ and $\sigma_{\theta}^{2}=$ $(5 \mathrm{deg})^{2}$ were used to perturb the theoretical sonar reading. Fig. 7 and Fig. 8 show the tracking of the distance and orientation of the wall in front of the AUV with and without the use of navigation information, respectively. In both the cases sonar-points and heading measurements are used for update during the tracking. From these figures, it is obvious that for both the cases the ground truth stays well within $2 \sigma$ bounds of the estimate. Although the tracking is smoother in the case where navigation is used. On the other side, the noise added in the range is significantly large $(30 \mathrm{~cm})$. Hence, if a $95 \%$ confidence level is used with such a noisy data, spurious measurements are also used to update the estimate which results in an erratic tracking behaviour. It has been observed that reducing the confidence level down to $60 \%$ makes the tracking smoother. At the same, for the case without navigation, we cannot afford to have a very low confidence as it reduces the number of updates and hence discardes a lot sonar data which is the only means of correcting the wall's relative distance and orientation.
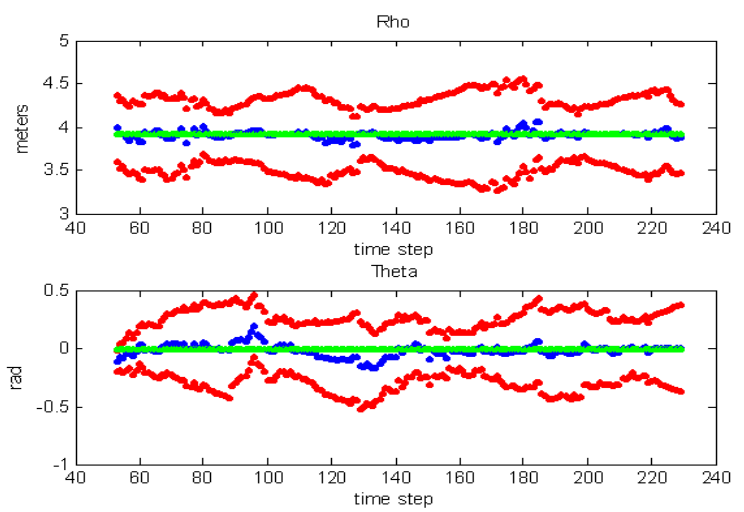

Fig. 7. Line Tracking with navigation. In red $2 \sigma$ bounds of the tracked blue line. Ground truth in green. (Synthetic Data, $\sigma_{\text {range }}=30 \mathrm{~cm}$, $\sigma_{\text {bearing }}=$ $5^{\circ}$, Sector Size $=90^{\circ}$, robot in Motion, $95 \%$ confidence)
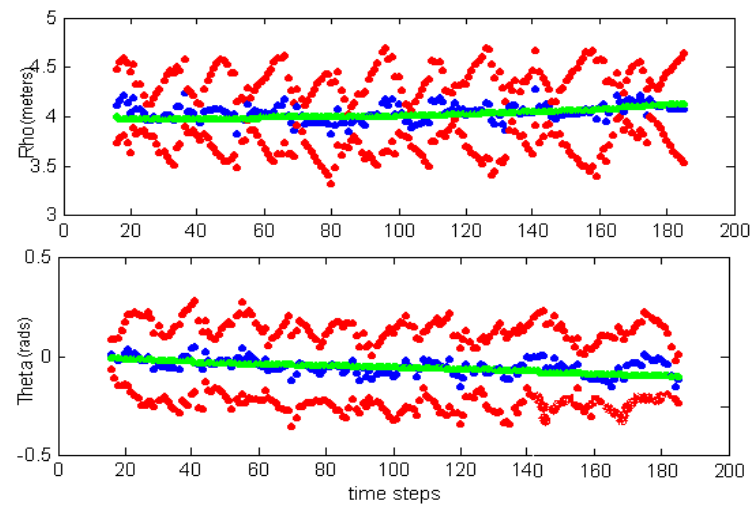

Fig. 8. Line Tracking without navigation. In red $2 \sigma$ bounds of the tracked blue line. Ground truth in green. (Synthetic Data, $\sigma_{\text {range }}=30 \mathrm{~cm}$, $\sigma_{\text {bearing }}=5^{\circ}$, Sector Size $=90^{\circ}$, robot in Motion, $95 \%$ confidence)

\section{B. Experiments}

Short duration experiments were conducted where the robot was facing a wall $\approx 4-5 m$ away with slight movements. The initial position of the wall in the robot frame was manually measured and used as the ground truth. 

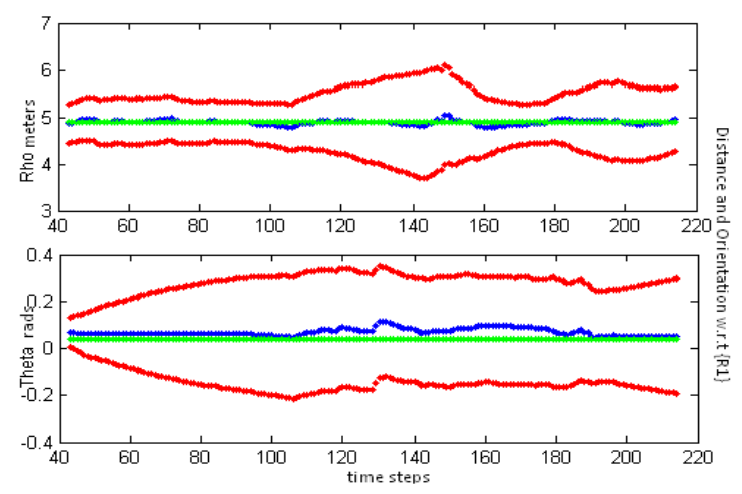

Fig. 9. Real Data, with Navigation, $95 \%$ confidence, robot in slow motion while facing a wall (at $90^{\circ}$ orientation in $\{\mathrm{W}\}$ )

As it is obvious from the Fig. 9, the tracking is very smooth as compared to simulated data because the robot movements are slower than those simulated and observed sonar noise is also comparatively less than the synthetic data noise. Moreover, the ground truth stays well within the $2 \sigma$ bounds. The ground truth in $\theta$ is more stable in the bounds as compared to the simulated cases because in reality the heading of the robot was more stable than that in the simulations.
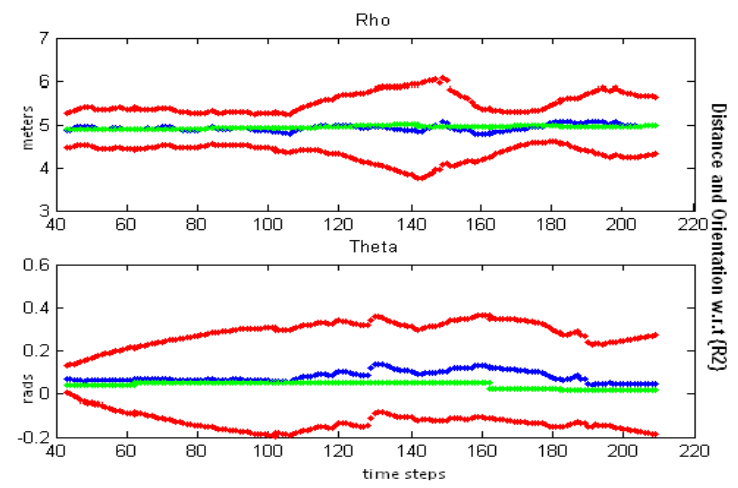

Fig. 10. Real Data, without Navigation, $95 \%$ confidence, robot in slow motion while facing a wall (at $90^{\circ}$ orientation in $\{\mathrm{W}\}$ )

Using the data gathered with the same experiment but using navigation information only to reference the ground truth to the robot frame while avoiding its use for the wall tracking (as the sonar data is not referenced to a specific reference frame in the update part of the EKF) (Fig. 10), still ground truth stays well within the $2 \sigma$ bounds of the estimate, more like the case with navigation with slightly more distortions in the estimate after time step $t=140$. As a consequence of not using navigation information, the change in the ground truth in $\theta$ can be observed.

\section{CONCLUSIONS}

In this work a method for detection and tracking of a line feature in the robot fixed frame, with application to dam wall inspection task, is developed. Depending on the characteristics of the robot motion, tracking can be achieved with or without navigation. If the robot moves fast or suddenly, for instance, when affected by strong perturbations, then navigation is necessary to avoid the motion induced distortion. In such a case, since the position estimate accumulates drift over time, the frame of reference must be changed periodically to cancel the undesired drift effects over long duration experiments. Nevertheless, if the robot motion is smooth, the wall tracking can be achieved without knowing the robot position. This is of particular interest when an ROV is used instead of an AUV, since ROVs commonly do not incorporate other navigation sensors than a heading sensor. With no position information from the navigation devices, the sonar data is the only source to extract relative position information. Hence, in this case, the position estimate of the wall with respect to the vehicle or vice versa is heavily dependent on the accuracy of sonar data . Furthermore, in this situation, the system cannot afford to discard a lot of sonar data by using a very strict confidence level and vehicle movements must be slow enough to allow the EKF estimate to gradually evolve. Still, working within most of these constraints is not very difficult. In fact, in scenarios such as wall survey of a dam, slow movement of the vehicle is a requirement of the inspection task. So, for such tasks, the method of tracking without navigation is quite suitable.

\section{ACKNOWLEDGMENTS}

The authors gratefully acknowledge the support of VIBOT Master's consortium and the reviewers' comments.

\section{REFERENCES}

[1] J. Lavalle, J. Cote, "Augmented reality graphic interface for upstream dam inspection", SPIE, pp. 33-39, December 1995.

[2] M. Poupart, et al., "Subacuatic inspections of EDF (Electricite de France) dams", OCEANS 2000. MTS/IEEE Conference and Exhibition, Volume:2, pp.939-942, Nov.2001.

[3] J. Batlle, T. Nicosevici, R. Garcia and M. Carreras, "ROV-Aided Dam Inspection: Practical Results", 6th IFAC Conference on Manoeuvring and Control of Marine Crafts, 2003.

[4] D. Ribas, N. Palomer, P. Ridao, M. Carreras and E. Hernandez, "ICTINEU ${ }^{A U V}$ Wins the first SAUC-E competition", IEEE Int. Conference on Robotics and Automation, Roma., Italy, April-2007.

[5] Stutters, L., Honghai Liu, Tiltman, C., Brown, D.J., "Navigation Technologies for Autonomous Underwater Vehicles", IEEE Transactions on Systems, Man, and Cybernetics, Part C: Applications and Reviews, vol.38, no.4, pp.581-589, July 2008

[6] Vaganay, J., Elkins, M.L., Willcox, S., Hover, F.S., Damus, R.S., Desset, S., Morash, J.P., Polidoro, V.C., "Ship hull inspection by hull-relative navigation and control," OCEANS, 2005. Proceedings of MTS/IEEE, pp. 761-766 Vol. 1, 2005

[7] Nguyen V., Gachter S., Martinelli A., Tomatis N., and Siegwart, "A comparison of line extraction algorithms using 2D range data for indoor mobile robotics", Auton. Robots 23, 2 (Aug. 2007), 97-111

[8] D. Ribas, P. Ridao, J. Neira, J.D. Tardos, "A Method of Extracting lines and their uncertainty from acoustic underwater images for SLAM", 6th IFAC Symp. on Inteli. Auton. Vehicles, Toulouse, France, Sep-2007.

[9] J.J. Leonard, H.F. Durrant-Whyte, "Directed Sonar Sensing for Mobile Robot Navigation", Kluwer Academic Publishers, Boston-1992.

[10] J.D. Tardos, J. Neira, P.M. Newman, and J.J. Leonard, "Robust mapping and localization in indoor environments using sonar data", Int. J. Robotics Research, 21(4), 2002.

[11] Ribas, D., Ridao, P., Tards, J. D., and Neira, J. "Underwater SLAM in man-made structured environments". Journal of Field Robot. 25, 11-12 (Nov. 2008), 898-921

[12] D. Ribas, J. Neira, P. Ridao, J.D. Tardos, "AUV Localization in Structured Underwater Environments using an A Priori Map", 7th IFAC Conference on Maneuvering and Control of Marine Crafts, Lisbon, Portugal, Sept-2006. 